\title{
Sowjetpropaganda und Animationsfilme
}

ULRICH SCHMID

Animationsfilme eignen sich besonders gut zur Implementierung bestimmter Kulturtechniken, die von jeder normativen Ideologie dringend benötigt werden. Alle Diktaturen müssen ihre eigene Ideologie inszenieren, um überhaupt ein Gesicht zu haben. Herfried Münkler hat die überzeugende These vertreten, dass die autoritäre politische Machtausübung über einen höheren unsichtbaren Anteil verfüge als die Demokratie. Deshalb müsse ein autoritärer Herrscher zu weitreichenden Visualisierungsstrategien greifen, sobald seine Sichtbarkeitsreserven aufgebraucht seien (Münkler 1995: 213-230). Ohne dauernde Visualisierung der Herrschaftsideologie droht sich die öffentliche Sphäre zu verselbstständigen - und in diesem Fall kann die autoritär definierte Wirklichkeitsdeutung zerfallen. Zygmunt Bauman (I995) hat den Hauptzweck der Modernisierungsdiktaturen des 20. Jahrhunderts in der Herstellung von „Eindeutigkeit“ erblickt. Allerdings ist dieses Ziel kaum je in einer Reinform erreicht worden. In der konkreten Ausgestaltung der Visualisierung von Herrschaft schleichen sich immer wieder subversive Tendenzen ein.

Diese These lässt sich exemplarisch an der sowjetischen Animationsfilmproduktion belegen. Die offizielle Sowjetkultur war lange Zeit von einer dichotomischen Weltsicht geprägt. Besonders deutlich lässt sich diese ideologische Grundlage an der ersten Verfassung der Sowjetunion aus dem Jahre I923 ablesen (Schmid 20I0: 43I-45I). Hier wird die paradiesische Sowjetunion den depravierten kapitalistischen Ländern gegenübergestellt:

Seit der Bildung der Sowjetrepubliken haben sich die Staaten der Welt in zwei Lager gespalten: das Lager des Kapitalismus und das Lager des Sozialismus. Dort, im Lager des Kapitalismus, herrschen nationale Feind schaft und Ungleichheit, Kolonialsklaverei und Chauvinismus, nationale Unterdrückung und Pogrome, imperialistische Grausamkeiten und Krie- 
ge. Hier, im Lager des Sozialismus, herrschen gegenseitiges Vertrauen und Friede, nationale Freiheit und Gleichheit, friedliches Zusammenleben und brüderliche Zusammenarbeit der Völker (Istorija sovetskoj konstitucii I9I7-I957: 226).

Bereits Lenin hatte den Film als die wichtigste aller Künste für die Bolschewiki bezeichnet (Gak I973). Sobald der technische Fortschritt es erlaubte, setzte die Sowjetführung ab I928 mobile Kinoanlagen ein, die auch die ländliche Bevölkerung erreichen sollten. Seit I932 war auch ein Kinozug unterwegs, der in verschiedenen Provinzstädten Halt machte und die neusten Produktionen der jungen sowjetischen Filmindustrie zeigte (Nembach 200I: 79, I83). Im Juni I936 wurde in Moskau das erste staatliche Animationsfilmstudio „Sojuzmul'tfil'm“ gegründet. Die meisten Animationsfilme gehören zum Genre des Märchens und weisen eine mehr oder weniger ausgeprägte politische Dimension auf. Das Märchen eignet sich wegen seiner starken moralischen Aufladung, der prototypischen Struktur und der eindimensionalen Charaktere gut für die narrative Umsetzung von Propaganda. Stalin war ein begeisterter Kinoliebhaber und schenkte der Filmproduktion große Aufmerksamkeit. In seinen letzten Lebensjahren gab er die Devise aus, in der ganzen Sowjetunion nur noch vier bis sechs Spielfilme pro Jahr zu drehen, dafür aber mit höchster Qualität ( Shaw / Youngblood 2010: 4I).

Nach dem Zweiten Weltkrieg herrschte in der Sowjetunion eine xenophobe Atmosphäre, die sich auch in den Filmsujets niederschlug (Graffy 2008: 27-46, 27). Wie wichtig die Filmindustrie war, zeigt die Tatsache, dass von I946 bis I953 ein eigenes Kinematografie-Ministerium existierte, das allerdings nach Stalins Tod direkt in ein neu gegründetes Kulturministerium überführt wurde. In den Ig6oer-Jahren profitierte die sowjetische Kinoindustrie vom Tauwetter und brachte auch gesellschaftskritische Filme hervor. In den I970er- und frühen I980er-Jahren verschärfte sich das ideologische Klima wieder - emblematisch für diese Entwicklung ist der bizarre Spielfilm Flug 222 ( I985) von Sergej Mikaèljan: Ein sowjetischer Balletttänzer springt während einer Tournee in New York ab, ohne seiner Frau etwas zu sagen. Diese und alle übrigen Passagiere im sowjetischen Flugzeug, das den Tänzer nach Hause bringen sollte, wollen jedoch nicht in den USA bleiben, sondern ins Arbeiterparadies zurückkehren. Die US-amerikanischen Behörden schikanieren die Menschen im Flugzeug, können jedoch den Sowjetpatriotismus nicht brechen.
Animationsfilme nehmen in der sowjetischen Kinoproduktion eine Sonderstellung ein. Hier überwiegt das satirische Genre, weil sich in Animationsfilmen groteske Körperdeformationen sehr gut darstellen lassen. Diese Eigenschaft kommt auch der ideologischen Indoktrination zugute: Durch die Karikierung der Figuren wird die Sympathie der Zuschauer ein deutig in eine bestimmte Richtung gelenkt. In den I940er- und I950er-Jahren wurde die sowjetische Animationsfilmproduktion sehr stark von Walt Disney beeinflusst. Wie das amerikanische Vorbild setzte „Sojuzmul'tfil'm“ eine plastische Bildsprache ein, in der die beiden Hauptkunstgriffe „stretching“ und „squeezing“ dominierten. Nach dem Zweiten Weltkrieg setzte der ideologische Kampf gegen die „Disneevščina“ ( „Disneytum“) ein, der bald darauf zu neuen Techniken wie etwa der Collage führte. Damit änderte sich auch das Genre: Neben dem dramatischen Kampf zwischen Gut und Böse waren nun auch tragische Sujets möglich, die für die Umsetzung ihrer ideologischen Absicht eine Goya-Ästhetik einsetzen.

Die erste „Sojuzmul'tfil'm“-Produktion, In Afrika ist es heiß (1936), visualisiert einen zentralen Pfeiler der Sowjetideologie, die Völkerfreundschaft. Tiere des Südens und des Nordens tauschen Geschenke (Speiseeis und Bananen) aus und stellen so ihre Verbundenheit unter Beweis. Der Animationsfilm Hier wird nicht gebissen ( I937) warnt in allegorischer Form vor Volksfeinden: Alle Tiere laufen Schlittschuh auf einem gefrorenen Weiher, der Wolf stellt sich freundlich und lädt die Häsin zu einem Spaziergang ein. Als der Wolf die Häsin angreift, sind zum Glück alle Tiere da und verjagen den Wolf mit Pauken und Trompeten. In diesem Sujet greift der Animationsfilm das stalinistische Paradigma der „Aufmerksamkeit“ auf: Jeder Sowjetbürger war verpflichtet, Saboteure, Schädlinge und Diversanten zu erkennen und zu denunzieren. Der Jäger Fëdor (I938) basiert auf einem burjatischen Märchen, das am 9. September I937 in der „Prav$d a$ “ abgedruckt worden war: Ein kleiner Junge verjagt die imperialistischen Japaner, die Sowjetrussland angreifen. Die Ausbeutung eines Jungen durch einen reichen Großgrundbesitzer im Film Das Märchen vom guten Umar (1938) weist bereits eine Paradoxie auf. Der kleine Umar wird von einem bösen Baj übervorteilt, Umar erhält für seine Herzensgüte einen Wunschring, mit dessen Hilfe er sich einen Palast wünscht. In der Exposition dieses Films gilt noch die klassische marxistische Gegenüberstellung von Ausbeutern und Proletariern. Am Ende wird der positive Held jedoch selbst reich und darf die Früchte seiner Arbeit genießen. An dieser Handlungsführung lässt sich die Verbürgerlichung der Sowjetkultur in den 
I930er-Jahren ablesen. Es war Stalin selbst, der sich in einer programmatischen Rede aus dem Jahr I93I verächtlich gegen die „Gleichmacherei“ wandte und eine leistungsabhängige Entlohnung der Staatsangestellten forderte (Mehnert I958). Konsequenterweise musste auch das Eigentum geschützt werden: Artikel Io der Stalinverfassung von I936 garantierte das persönliche Eigentum und sogar das Erbrecht:

Das Recht des persönlichen Eigentums der Bürger an ihren selbst erarbeiteten Einkünften und Ersparnissen, am Wohnhaus und an der häuslichen Nebenwirtschaft, an den Gegenständen der Hauswirtschaft und des Haushalts, an Gegenständen des persönlichen Bedarfs und Komforts ebenso wie das Erbrecht am persönlichen Eigentum der Bürger, werden durch das Gesetz geschützt ( Istorija sovetskoj konstitucii 1957: 341 ).

Während des Zweiten Weltkriegs wurden Animationsfilme als animierte Poster eingesetzt. In Mikrosujets wurde den sowjetischen Zuschauern gezeigt, was bei einer Eroberung der Sowjetunion durch Hitler geschehen würde. Auch hier mussten die Regisseure einen schwierigen Spagat bewältigen. Auf der einen Seite musste Hitler als gefährlicher Gegner porträtiert werden, auf der anderen Seite durfte kein Zweifel an einem sowjetischen Sieg aufkommen. Im Jahr I94I, nach dem deutschen Überfall auf die verbündete Sowjetunion, produzierte „Sojuzmul'tfil'm“ eine eigene Wochenschau mit sogenannten „Politplakaten“. Die Darstellung Hitlers folgte ziemlich genau politischen Karikaturen aus der Printpresse. Der Film Was Hitler will präsentiert sich als Verfilmung eines TASS-Fensters, die ein wichtiges Instrument der Kriegspropaganda darstellten. Die TASS-Fenster waren politische Cartoons, die zentrale politische Aussagen auf einen einfachen grafischen Nenner brachten. Was Hitler will war ein Plakat (I94I) von Michail Čeremnych mit sechs einfachen Szenen, in denen die Nazi-Aggression angeprangert wurde (Waschik / Baburina 2003: 2II). Der Film griff diesen Plot auf und zitierte in seiner visuellen Umsetzung bekannte Gemälde aus der russischen Kunstgeschichte. So besteigt etwa Hitler einen Berg von Totenschädeln, der bereits ein prominentes Motiv in Vasilij Vereščagins Gemälde Apotheose des Kriegs ( I87I) darstellt.
Abbildung 1: Hitler auf einem Berg von Schädeln seiner Opfer

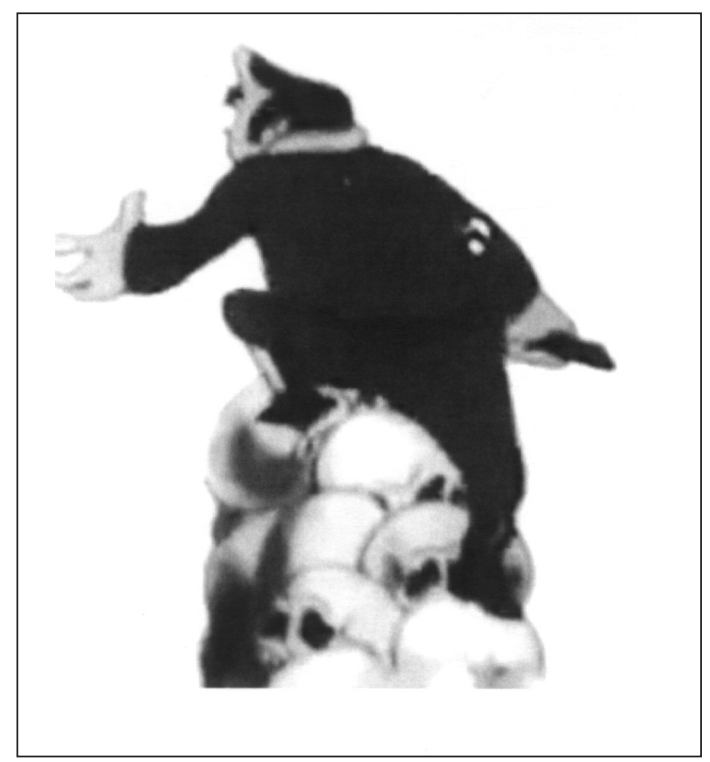

Quelle: Was Hitler will ( 1941: 8':03“)

Abbildung 2: Vasilij Vereščagin: Apotheose des Kriegs (1871)

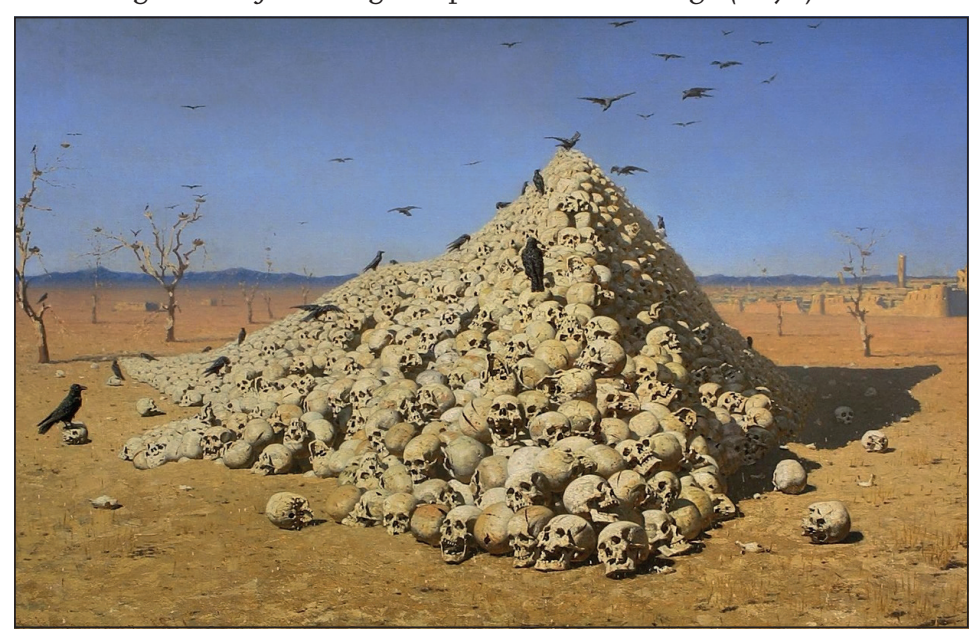

Quelle: Verešeagin (1871) 
Der Krieg stellte nach dem Zeugnis von Joseph Brodsky für viele Künstler und Schriftsteller fast eine Erleichterung dar, weil man endlich menschliches Leiden und negative Erscheinungen benennen konnte und nicht ausschließlich den staatlich verordneten Aufbauoptimismus predigen musste (Brodsky I985: 543-544). Nach dem Krieg verschärfte Stalin die Gangart jedoch wieder. Die veränderte ideologische Situation spiegelte sich etwa im Animationsfilm Fedja Zajcev ( I947). Der kleine Fedja malt in seinem Klassenzimmer ein Männchen an die Wand. Er traut sich jedoch nicht, seinen Fehltritt zu gestehen und schiebt die Schuld auf einen Klassenkameraden $\mathrm{ab}$. $\mathrm{Zu}$ Hause wenden sich alle Spielzeuge und die Helden seiner Lieblingsbücher von ihm ab. Fedja gesteht seine Schuld. Dieser Film stellt eine deutliche Visualisierung stalinistischer Bekenntnispraktiken dar. Jedes Parteimitglied hatte das Verfahren von Kritik und Selbstkritik zu durchlaufen. Schlimmer noch als mögliche Fehler war dabei das Verschweigen eines Fehlers. Im Film Die fremde Stimme (1949) wird der zwar attraktive, aber verderbliche Einfluss des Westens dargestellt: Die Tiere im Wald lauschen dem Gesang der Nachtigall. Da taucht eine Elster auf, die aus dem Ausland nach Russland geflogen ist. Sie erklärt den Nachtigallengesang für veraltet und gibt ein Konzert. Die Tiere sind zunächst fasziniert vom unbekannten Gesang der Elster, merken aber schließlich, dass dies nichts für sie ist.

Nach Stalins Tod verschiebt sich der ideologische Akzent auf den Gegensatz Kommunismus-Kapitalismus. Die goldene Antilope (I954) basiert auf einem indischen Märchen und erzählt die Geschichte eines Waisenjungen, der eine fliehende Antilope vor Jägern rettet. Die Antilope kann mit ihren Hufen Gold erzeugen. Als ein reicher Raja davon erfährt, nimmt er den Waisenjungen gefangen, der aber bald von der Antilope befreit wird. Der ideologische Witz der Handlung besteht darin, dass der Junge das Gold der Antilope gar nicht braucht, während der Raja die unendliche Kapitalakkumulation als Selbstwert betrachtet. Er sagt zur Antilope: „Dummes Tier! Gold kann es nie genug geben!“ In ironischer Umkehrung dieses Credos kommt der Raja schließlich in einer Flut von Goldmünzen um.
Abbildung 3: Die goldene Antilope (1954)

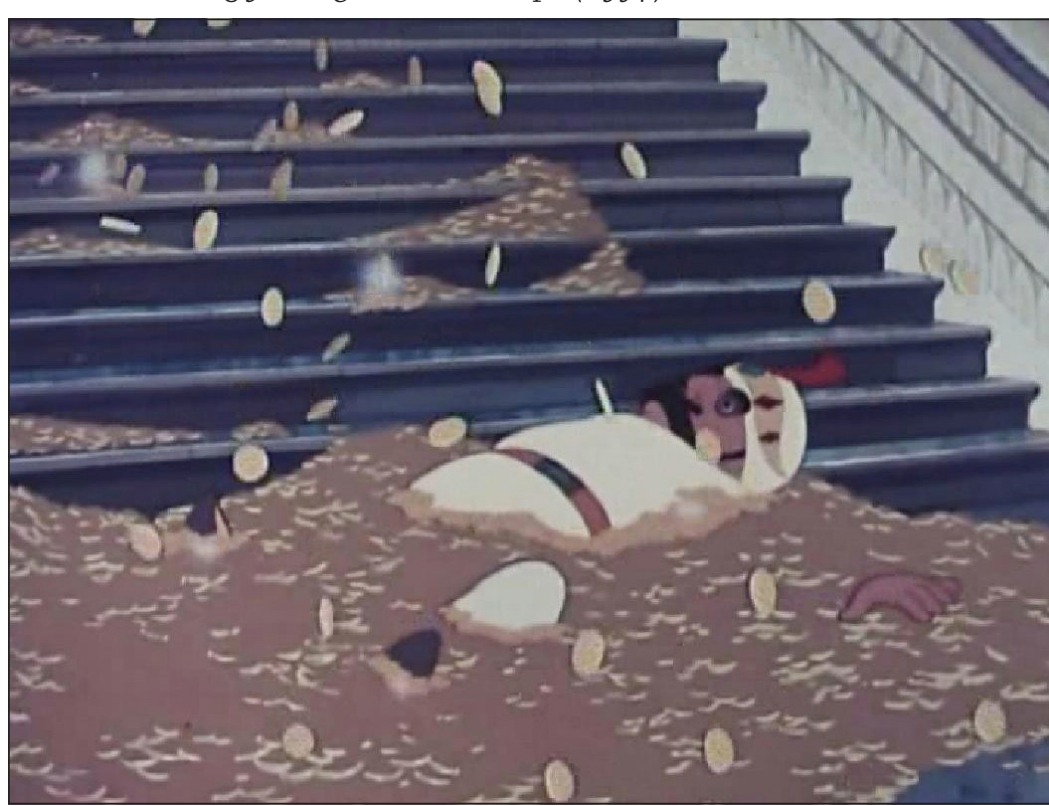

Quelle: Atamanov (1954: 29':02“)

In den I96oer-Jahren kommt es in der Sowjetunion zu einer Reihe von Literaturadaptionen, die Gegenstücke zu einer Disney-Verfilmung bilden. I967 wurde die erste Serie Rakša der insgesamt fünfteiligen Maugli-Verfilmung (I967-I97I) von Roman Davydov produziert. Während Disneys Dschungelbuch ( 1967 ) ganz als Musikkomödie aufgebaut ist, folgt die sowjetische Version dem Original von Rudyard Kipling deutlicher. Maugli ist ein Held, der sich einer feindlichen Umwelt stellen und um sein Leben kämpfen muss.

I969 folgte eine sowjetische Verfilmung von Alan Alexander Milnes Winnie the Pooh. Damit nahm die Sowjetunion ein Erfolgsrezept auf, dass die Disney- Studios mit dem Kurzfilm Winnie the Pooh and the Honey Tree ( I966) begonnen hatten. I968 erhielt die Fortsetzung Winnie Pooh and the Blustery Day sogar einen Oscar. 1974 erschien Winnie Pooh and Tigger, too!, I977 wurde sogar ein abendfüllender Animationsfilm mit dem Titel The Many Adventures of Winnie the Pooh produziert.

Im Gegensatz zur Disney-Version stattete der Regisseur Fëdor Chitruk seinen Protagonisten mit einem komplexen Charakter aus, der sowjet- 
ische „Vinni Puch“ kann auch traurig sein und sich von seinen Freunden zurückziehen. Eine weitere Veränderung betrifft Christopher Robin, der in der sowjetischen Version konsequent durch Piglet ersetzt wird. Chitruks Verfilmung erreicht eine höhere ästhetische Autonomie, weil sich alle Abenteuer innerhalb der Tierwelt von Vinni Puch abspielen. Ein „stand in“ für den kindlichen Zuschauer, wie ihn Christopher Robin darstellt, fehlt - der Junge wurde als Gesprächspartner für den Bären konsequent durch „Piglet/Pjatačok“ ersetzt. Der erste Vinni Puch-Film war so erfolgreich, dass in den Jahren I97I und 1972 zwei weitere Filme folgten.

Sowohl Maugli als auch Vinni Puch haben keine explizite politische Aussage. Maugli folgt jedoch den Stilprinzipien des sozialistischen Realismus und zeigt einen positiven Helden, der sich gegen negative Figuren durchsetzen muss. Dabei folgt die Handlung dem prototypischen Plot, wie ihn Katerina Clark für den Produktionsroman beschrieben hat: Der Held kommt in einem Mikrokosmos an, erhält dort eine Aufgabe, muss verschieden Prüfungen bestehen, scheitert beinahe, erhält während eines Treffens mit einem Mentor neue Kräfte, durchlebt eine Initiation und erfüllt schließlich seine Aufgabe (Clark 2000: 25I-266).

I968 entstand die politische Parabel Die Glasorgel, die allerdings von der Zensur zurückgehalten wurde. Der Film arbeitet mit zahlreichen Anspielungen auf klassische Kunstwerke (im Abspann wird mit einem Zwischentitel sogar explizit auf diese Besonderheit hingewiesen). In einer utopischen Stadt regiert der gelbe Teufel, das Geld. Ein Künstler besitzt eine Glasorgel, die alle Menschen glücklich macht und in ihnen edle Gefühle weckt. Allerdings wird der Künstler verhaftet, seine Glasorgel wird zerstört. Die Vertreter der Macht sind Melonenträger im Stil René Magrittes oder gesichtslose Schergen, die den entindividualisierten Bauern im Spätwerk von Kazimir Malevič ähneln. Darauf verwandeln sich die Bewohner der Stadt in Monster und Raubtiere, die Gestalten von Hieronymus Bosch, Francisco Goya, Giuseppe Arcimboldo und Pieter Breughel nachempfunden sind. Einem jungen Mann gelingt es, eine neue Glasorgel zu beschaffen - darauf verwandelt sich die graue Gefängnissiedlung in eine Renaissancestadt mit Figuren aus dem Werk von Albrecht Dürer, Pinturicchio und El Greco.
Abbildung 4: Der junge Künstler aus der Glasorgel (1968)

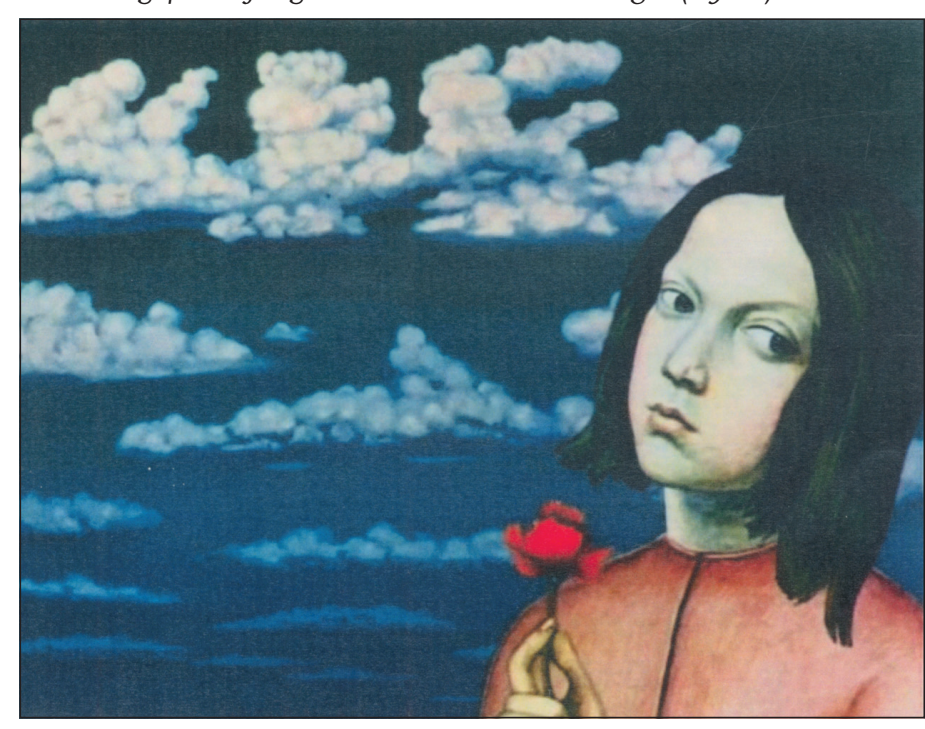

Quelle: Chržanovskij (1968: 10’:07“)

Abbildung 5: Der goldene Teufel aus der Glasorgel (1968)

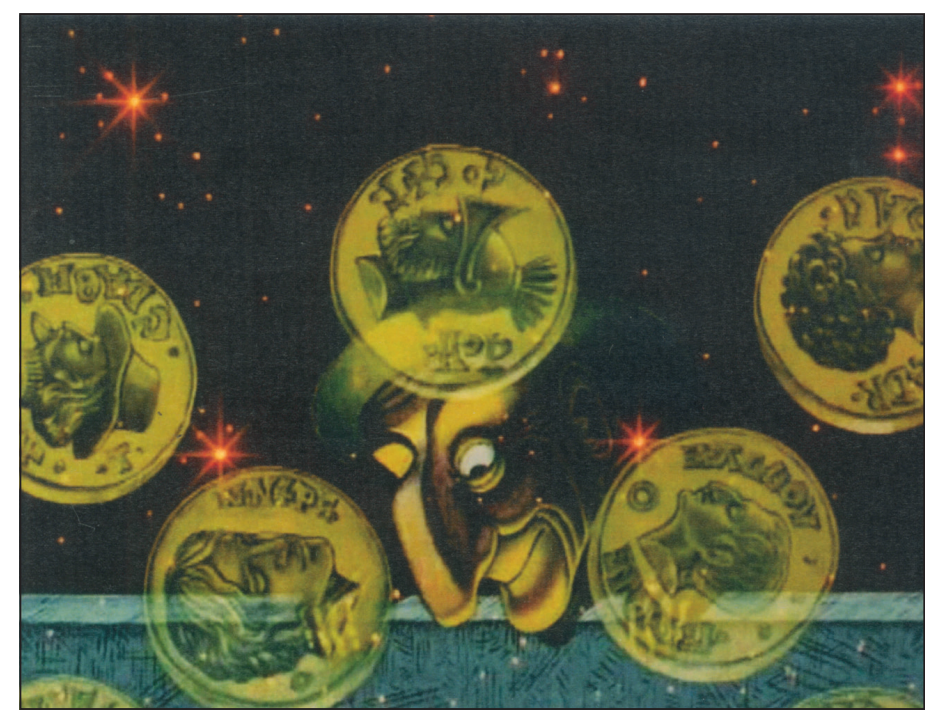

Quelle: Chržanovskij ( 1968: 6':16“) 
Die Stadt befindet sich unter dem Fluch des „gelben Teufels“ - hier kommt die übliche antikapitalistische Kritik zum Tragen. Der Animationsfilm ist nicht frei von antisemitischen Untertönen - die Fratze des geldgierigen Bösewichts folgt den Darstellungskonventionen jüdischer Hassfiguren.

Eine wichtige Rolle spielt in diesem Film über das Verhältnis von Macht und Kunst die Musik, die von Alfred Schnittke komponiert wurde. Das Leitmotiv der Glasorgel besteht aus der Tonfolge B-A-C-H, die auch von Johann Sebastian Bach als musikalische Signatur in seinen Werken eingesetzt wurde. I968 war ein schwieriges Jahr in der sowjetischen Kulturpolitik - nach dem Tauwetter der frühen ig6oer-Jahre, das etwa auch die Publikation von Aleksandr Solženicyns Lagererzählung Ein Tag im Leben des Ivan Denisovič ermöglicht hatte, kühlte sich das Klima nach dem Sturz Chruščevs deutlich ab. I966 wurden die Schriftsteller Andrej Sinjavskij und Julij Danièl' zu mehrjährigen Lagerstrafen verurteilt. Auch um Solženicyn zog sich die Schlinge zu - er konnte nicht mehr publizieren; das Romanmanuskript Der erste Kreis der Hölle wurde beschlagnahmt. Die Produzenten der Glasorgel mussten deshalb zu Beginn des Films auf einem Zwischentitel eine ideologische Erklärung einschalten: „Obwohl der Film einen fantastischen Charakter trägt, wollten die Autoren an die hemmungslose Unpersönlichkeit, die polizeiliche Willkür, die Vereinzelung und Verwilderung der Menschen in der heutigen bourgeoisen Gesellschaft erinnern." Allerdings verhalf auch diese aufgesetzte Interpretation dem Film nicht zu einer öffentlichen Präsentation.

In den I970er-Jahren experimentierte „Sojuzmul'tfil'm“ mit einer neuen Ästhetik, die vor allem von der US-amerikanischen Pop-Art inspiriert war. Interessant ist der Animationsfilm Ave Marija aus dem Jahr I972, in dem der Vietnamkrieg angeprangert wird. Der Film ist mit Schuberts Kunstlied unterlegt und zeigt in erstaunlicher Vorwegnahme einer Schlüsselszene aus Francis Ford Coppolas Apocalypse Now" (1979) den Helikopterangriff auf ein vietnamesisches Dorf. Dabei kommen Frauen und Kinder gewaltsam ums Leben. (Bei Francis Ford Coppola ist diese Szene mit Richard Wagners Walkürenritt unterlegt.)
Abbildung 6: Der Angriff der Helikopter in Ave Marija (1972)

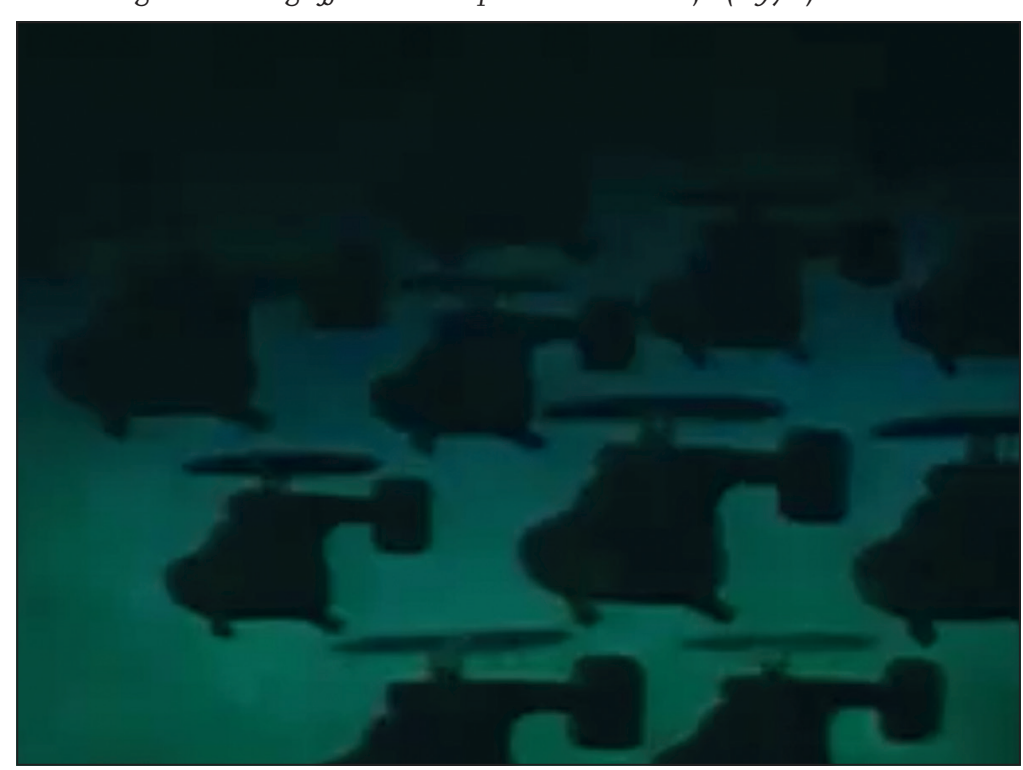

Quelle: Ivanov-Vano: Ave Marija ( 1972: 2':38“)

Abbildung 7: Der arbeitslose Amerikaner in Shooting Range (1979)

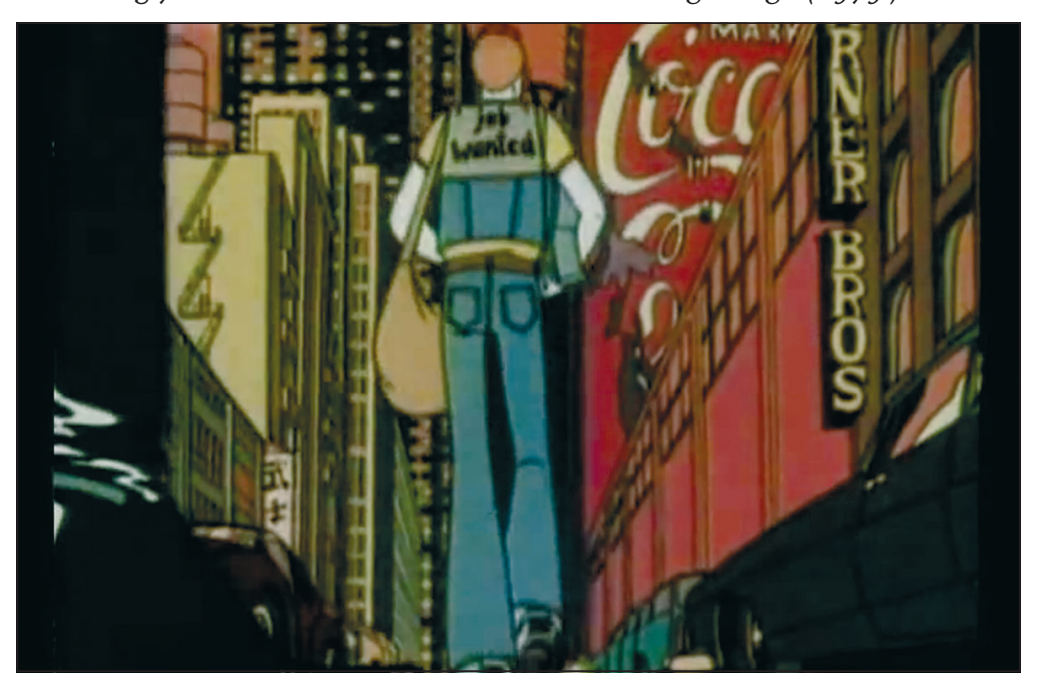

Quelle: Tarasov ( 1979: 3':58“) 
I979 entstand Tir (The Shooting Range), der das angeblich menschenverachtende Leben in den USA anprangert. Ein junger Arbeitsloser wird von einem skrupellosen Kapitalisten als lebendiges Ziel in einem New Yorker Schießstand engagiert. Der Junge verliebt sich in eine Leidensgenossin; sie bekommen ein Kind - der Schießbudenbesitzer kündigt darauf als besondere Attraktion ein Kind als Zielscheibe an.

Der Animationsfilm ist ganz in der US-amerikanischen Pop-Ästhetik gehalten, die als künstlerischer Stil gleichzeitig kritisiert und instrumentalisiert wird. Dasselbe gilt für die musikalische Untermalung, die während der Titelszene aus schrillen Jazzklängen besteht. Später verwandelt sich die Musikbegleitung in anspruchsvollen Jazz. Damit gelingt es dem Regisseur Vladimir Tarasov, eine in der Sowjetunion verpönte Musikrichtung auf politisch korrekte Weise in seinen Film zu integrieren: Zu Beginn wird die musikalische Falschheit des Jazz programmatisch signalisiert, später kann die Musik ihre ästhetische Autonomie entfalten.

Der Film beginnt mit einem visuellen Zitat - er zeigt eine schematische Luftaufnahme von New York, die ganz in Rosa getaucht ist. Damit verweist Tarasov auf die Ouvertüre von Robert Wises und Jerome Robbins' Verfilmung der West Side Story ( I96I), in der die Wolkenkratzer von Manhattan in einer monochrom wechselnden Verfremdung gezeigt werden.

Auch die Figuren aus Walt Disneys Animationsfilmen sind in Tarasovs Film vertreten. Die Glücksvision der beiden Liebenden wird durch den Einsatz des Elefanten Dumbo, der sieben Zwerge und des Hasen aus Bambi visualisiert.

Die Darstellung der Freundin des Protagonisten zitiert ebenfalls ein prominentes Vorbild: die weiblichen Porträts von Roy Lichtenstein.
Abbildung 8: Eingangssequenz von Shooting Range (1979)

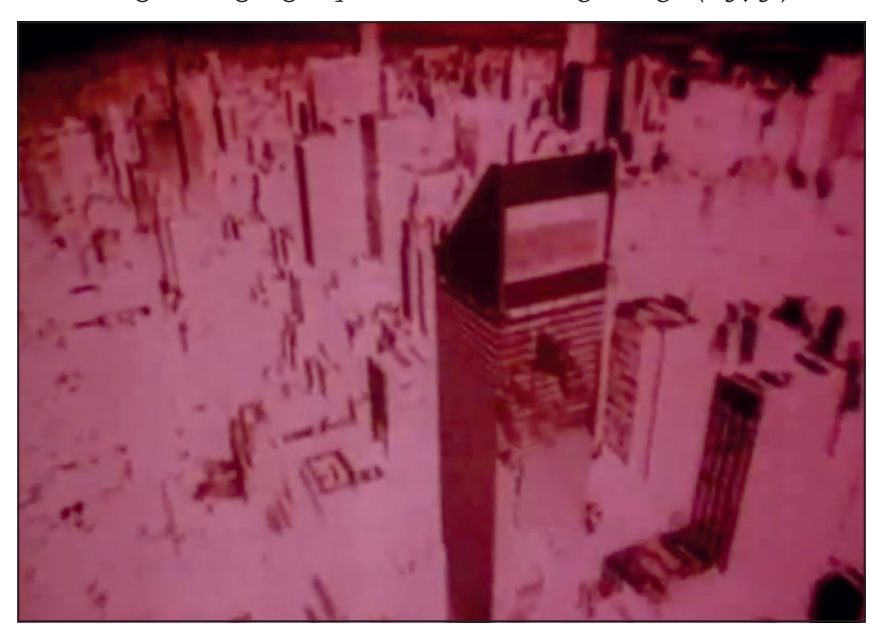

Quelle: Wise/Robbins ( 1961: 0':30“)

Abbildung 9: Die Freundin in Shooting Range (1979)

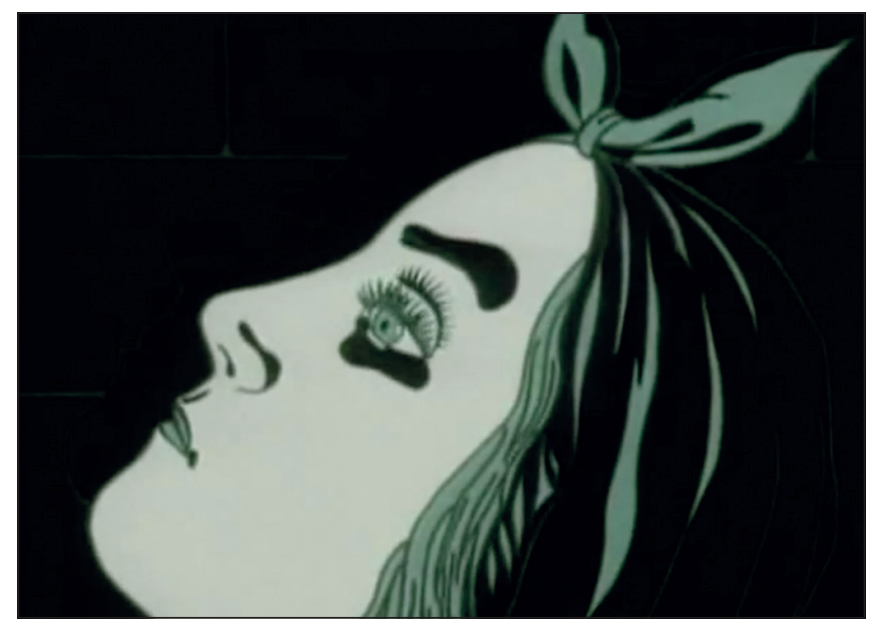

Quelle: Tarasov ( 1979: 9':54“) 
Auch die Ästhetik von Keith Haring ist in Tarasovs Animationsfilm präsent:

Abbildung 10: Die tanzenden Liebenden in Shooting Range (1979)

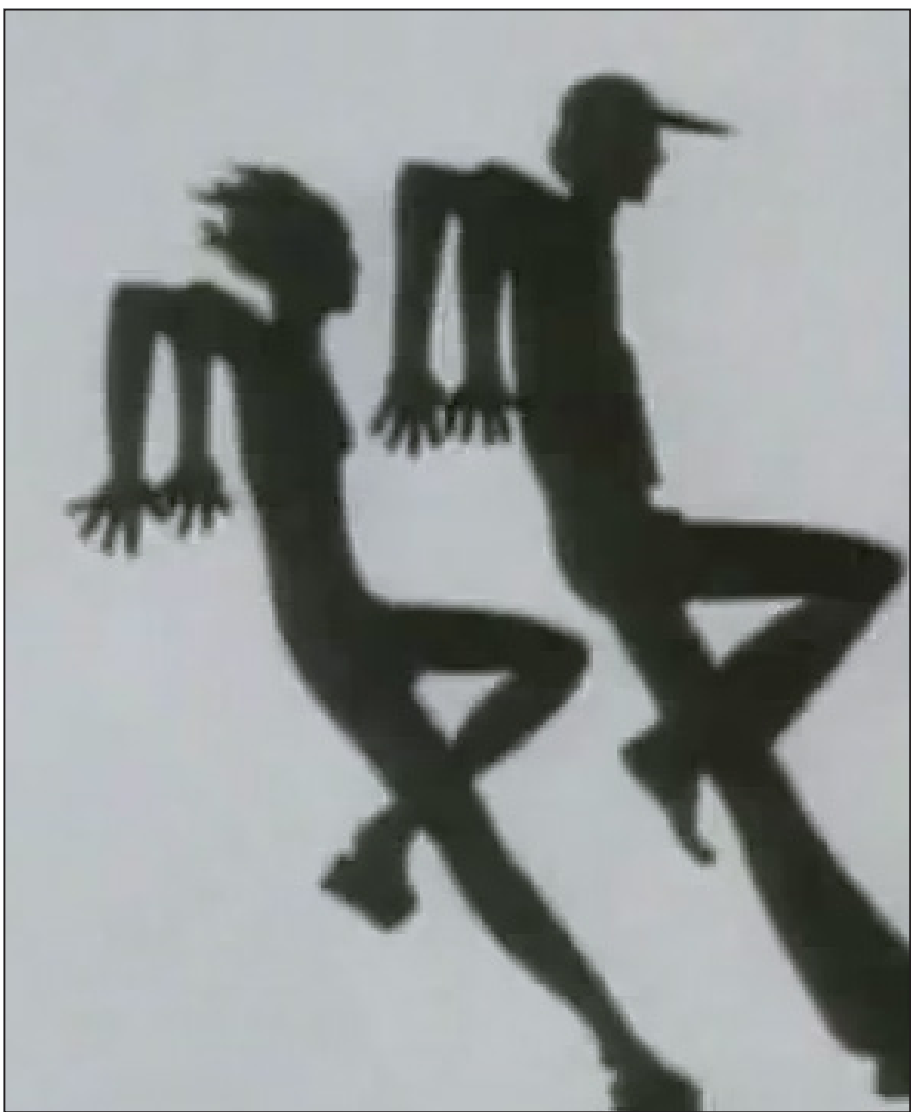

Quelle: Tarasov (1979: 11':32“)

Schließlich greift Tarasov jedoch auch auf den klassischen sowjetischen Fundus der Kapitalismuskarikatur zurück. Der Bösewicht befindet sich völlig außerhalb der dominanten Pop-Ästhetik des Films:

\section{Abbildung 11: Der Ausbeuter in Shooting Range (1979)}

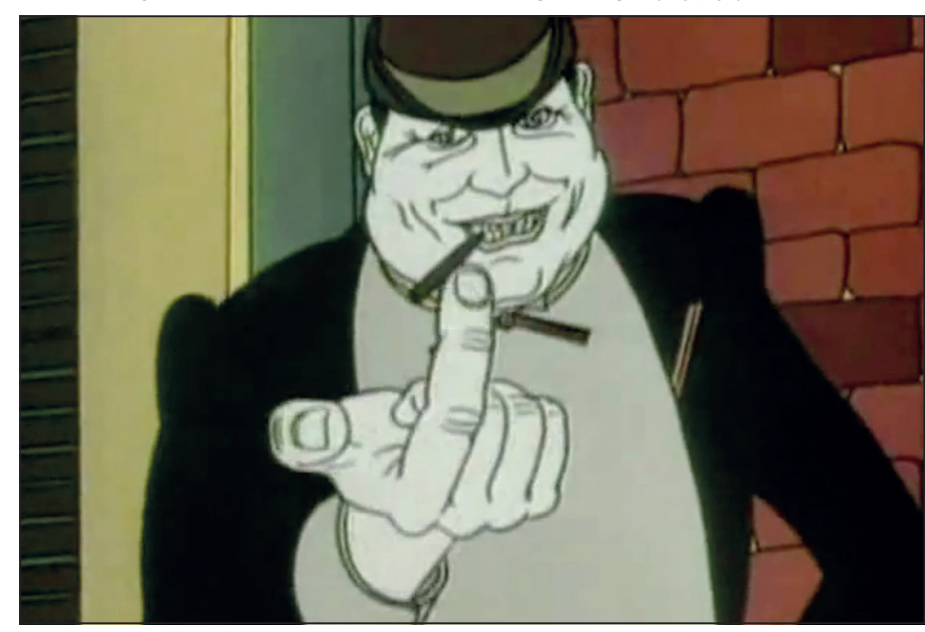

Quelle: Tarasov ( 1979: 4':06“)

Tarasovs Tir zeigt deutlich, dass die Herstellung von „Eindeutigkeit“ in Baumans Sinn in der späten Sowjetzeit höchstens noch ein Oberflächenphänomen darstellt. Die Visualisierung des US-amerikanischen Kapitalismus folgt den veralteten Modellen der Sowjetkarikatur aus den zwanziger und dreißiger Jahren. Die Darstellung der amerikanischen Jugendkultur hingegen bedient sich der Bildsprache der Pop-Ästhetik. Die jungen, arbeitslosen Amerikaner, die Opfer der Kapitalisten werden, sind in Tarasovs Film Sympathieträger, mithin ist auch die zitierte Ästhetik positiv konnotiert. Letztlich stellt Tir den interessanten Versuch dar, eine neue, moderne Visualisierung für eine alte Kapitalismuskritik zu finden.

Die sowjetischen Animationsfilme bemühten sich zumindest oberflächlich, ideologische Eindeutigkeit herzustellen. Eine traditionell marxistische Kapitalismuskritik findet sich in den meisten Filmen. Allerdings gerät die Visualisierung der Ideologie bereits bei der Darstellung der Bösewichte in ein Dilemma. Sehr oft zeichnet sich der Ausbeuter in den Animationsfilmen durch hohe Gewaltbereitschaft und Zynismus aus - damit rückt er in der Wahrnehmung der sowjetischen Zuschauer schnell in die Nähe der Parteinomenklatura. Möglicherweise liegt in dieser Besonderheit der Grund für die häufige Wahl exotischer Settings: Wenn das Böse in einer ganz anderen Kultur (Deutschland, Indien, USA oder einer Fantasie- 
welt) angesiedelt ist, kann das vertraute sowjetrussische Herrschaftsgefüge nicht direkt damit identifiziert werden. Diktatur und Ausbeutung sind dann fremde Phänomene in einer konstruierten Welt, die auf dramatische Weise erst zum sozialistischen Glück finden muss, das dem Anspruch nach bereits in der UdSSR vorhanden ist - noch I96I versprach Nikita Chruščev vollmundig, dass der Kommunismus im Jahr I980 in der Sowjetunion erbaut sein werde (Tompson I997: 238).

Die starke Popularität der Animationsfilme in der Sowjetunion lässt indes darauf schließen, dass sich das breite Publikum von solchen ideologischen Volten nicht beirren ließ. Die satirisch überzeichnete Welt der Animationsfilme hatte einen höheren Wirklichkeitsgehalt als die sozrealistischen Filme, die angeblich die Lebenswelt der Sowjetbürger auf gültige Weise nachzeichneten. Die Absurdität und die Entmündigung der individuellen Existenz in der Sowjetunion konnten viel besser durch die „Märchen für Erwachsene“ in den Animationsfilmen eingefangen werden als durch offizielle Kunstprodukte. Außerdem verzichteten viele Zeichentrickfilme in den späten Sowjetjahren auf Dialoge, um nicht mit der Zensur in Konflikt zu geraten. Deshalb befinden sich Animationsfilme in der Hierarchie der Künste bis heute in Russland viel höher als im Westen. Eine zusätzliche Konsekration erfuhren einige sowjetische Zeichentrickfilme durch die Mitarbeit berühmter Komponisten wie Dmitrij Šostakovič oder Alfred Schnittke.

Die visuelle Kultur der sowjetischen Animationsfilme emanzipierte sich am Ende der Stalinzeit von der Disney-Ästhetik und vollzog die Wende zu einer autonomen künstlerischen Existenz, die sich an der prominenten Kinderbuchproduktion sowjetischer Zeichenkünstler orientiert. Damit kommt den Animationsfilmen im Kontext der komplexen Sowjetkultur nicht nur eine wichtige ideologiekritische, sondern auch innovative ästhetische Funktion zu.

\section{LITERATURVERZEICHNIS}

Bauman, Zygmunt ( I995): Moderne und Ambivalenz. Das Ende der Eindeutigkeit. Frankfurt a. M.: Fischer Verlag.

Brodsky, Joseph (1985): „Literature and War. A Symposium: The Soviet Union“, in: The Times Literary Supplement vom I7.5.I985.

Clark, Katerina (2000): The Soviet Novel. History as Ritual. Bloomington: Indiana Univ. Press.

Gak, Aleksandr Mikhaı̌lovich (Hg.) (I973): Samoe važnoe iz vsech iskusstv. Lenin o kino. Sbornik dokumentov i materialov, Moskva.

Graffy, Julian (2008): „Scant Sign of Thaw. Fear and Anxiety in the Representation of Foreigners in the Soviet Films of the Khrushchev Years“, in: Stephen Hutchings: Russia and its Other(s) on Film. Screening Intercultural Dialogue. New York: Basingstoke, S. 27-46.

Istorija sovetskoj konstitucii. Sbornik dokumentov I9I7-I957 (I957). Moskva.

Nembach, Eberhard (200I): Stalins Filmpolitik. Die Reorganisation der sowjetischen Filmindustrie I929-38. Von liberaler Marktökonomie und kreativer Vielfalt zur zentral und monopolistisch gesteuerten Propagandamaschinerie. Diss. Bonn.

Mehnert, Klaus (I958): Der Sowjetmensch. Versuch eines Porträts nach zwölf Reisen in die Sowjetunion I929-I957, Stuttgart: Deutsche Verlags-Anstalt.

Münkler, Herfried (I995): Die Visibilität der Macht und die Strategien der Machtvisualisierung, in: Gerhard Göhler (Hg.), Macht der Öffentlichkeit - Öffentlichkeit der Macht, Baden-Baden: Nomos Verlag, S. $213-23 \mathrm{I}$.

Schmid, Ulrich (2010): „Constitution and narrative. Peculiarities of rhetoric and genre in the foundational laws of the USSR and the Russian federation", in: Studies in East European Thought 62, S. 43I-45I.

Shaw, Tony / Youngblood Denise (2010): Cinematic Cold War. The American and Soviet Struggle for Hearts and Minds. Kansas: University Press of Kansas.

Tompson, William J. (I997): Khrushchev: A Political Life, New York: St. Martin's Press.

Waschik, Klaus / Baburina, Nina (2003): Werben für die Utopie. Russische Plakatkunst des 20. Jahrhunderts. Bietigheim-Bissingen: edition tertium. 


\section{AbBiLdUngsVerzeICHNIS}

Abbildung I: Was Hitler will (I94I).

(http://www.youtube.com/watch?v=FRcBt904OJo).

Abbildung 2: Vereščagin, Vasilij ( I871): Apotheose des Kriegs.

(http://ru.wikipedia.org/wiki/Файл:Apotheosis.jpg).

Abbildung 3: Atamanov, Lev (I954): Die goldene Antilope. (http://www.youtube.com/watch?v=dqTdus3smgo).

Abbildungen 4 und 5: Chržanovskij, Andrej (I968): Die Glasorgel (http://www.youtube.com/watch?v=dYzoxck_NYQ).

Abbildung 6: : Ivanov-Vano, Ivan (I972): Ave Marija (http://www.youtube.com/watch?v=dl43n87kS7Q).

Abbildungen 7, 9, Iо und Ir: Tarasov, Vladimir ( I979): Tir (http://www.youtube.com/watch?v=N9uPQH_L9L4).

Abbildung 8: Wise, Robbins (I96I): West Side Story.

(http://www.youtube.com/watch?v=upHdYC6qWJo).

Die Videos wurden am 25. 7. 20I2 zuletzt abgerufen. 